Abstract 80 Table 1 Baseline characteristics of UK $(n=2346)$ vs. rest of the CLARIFY ( $n=30,555)$ population

\begin{tabular}{llll}
\hline $\begin{array}{l}\text { Baseline } \\
\text { characteristics }\end{array}$ & $\begin{array}{l}\text { UK } \\
\text { population \% }\end{array}$ & $\begin{array}{l}\text { Rest of CLARIFY } \\
\text { population \% }\end{array}$ & $\begin{array}{l}\text { p } \\
\text { value }\end{array}$ \\
\hline Male gender & 76.0 & 77.8 & 0.026 \\
History of MI & 65.1 & 59.5 & $<0.001$ \\
History of PCI & 41.4 & 59.9 & $<0.001$ \\
History of CABG & 27.3 & 23.2 & $<0.001$ \\
History of heart failure & 4.8 & 15.8 & $<0.001$ \\
Any antiplatelets & 91.7 & 95.3 & $<0.001$ \\
Statin use & 85.7 & 82.6 & $<0.001$ \\
ACEi/ARB use & 74.8 & 76.4 & 0.079 \\
Beta blocker use & 65.6 & 76.0 & $<0.001$ \\
\hline
\end{tabular}

Methods We carried out a retrospective analysis of sequential patients referred to the RACC with chest pain over a one month period. Data was collected for investigations performed, need for revascularisation, re-presentations and adverse events over 3 months of follow up. In addition to this, we reviewed the admission notes to evaluate what alternative management pathways may have been used in order to assess the number of overnight admissions, outpatient appointments or GP services that were saved.

Results 56 patients were seen in the clinic in the one month analysed. Of the 56 patients, $25(44.6 \%)$ were discharged directly from RACC with no investigations, 4 (7.1\%) had no investigations but had cardiology clinic follow-up, and 27 $(48.2 \%)$ required further cardiac investigations. 23 of this 27 $(85.2 \%)$ had invasive tests, $3(11.1 \%)$ had non-invasive and 1 had both (3.7\%). 4 of these were found to be abnormal and subsequently 1 was referred for coronary artery bypass grafting. The remainders were managed medically.

Re-presentations were few, with 1 patient attending ED over a weekend whilst waiting for the appointment with further troponin negative chest pain. There were 3 re-presentations within 3 months following RACC review. Of these, 1 admission was due to a headache and syncope, one was due to angina whilst waiting for coronary angiography and the third was due to further troponin negative chest pain. There were no adverse events.

It was estimated that 33 overnight admissions were avoided. Furthermore, 49 of the patients referred may

Abstract 80 Table 2 Outcomes at 2 year follow up for UK vs. rest of the CLARIFY population

\begin{tabular}{llll}
\hline Outcome & $\begin{array}{l}\text { UK } \\
\%\end{array}$ & $\begin{array}{l}\text { Rest of CLARIFY } \\
\text { population } \%\end{array}$ & $\begin{array}{l}\text { Hazard ratio }(95 \% \text { Confidence } \\
\text { interval); } \mathbf{p} \text { value }\end{array}$ \\
\hline $\begin{array}{l}\text { All cause } \\
\text { mortality }\end{array}$ & 3 & 3 & $0.96(0.75,1.23) ; 0.76$ \\
$\begin{array}{l}\text { Cardiovascular } \\
\text { death }\end{array}$ & 1.3 & 1.5 & $0.89(0.62,1.28) ; 0.52$ \\
$\begin{array}{l}\text { MI } \\
\begin{array}{l}\text { Non-fatal } \\
\text { stroke }\end{array}\end{array}$ & 1.3 & 0.7 & $1.56(1.19,2.03) ; 0.0012$ \\
\hline
\end{tabular}

alternatively have been managed via the primary care fast access chest pain service. This would have required a GP appointment and referral therefore involving significant primary care resources.

Conclusions The RACC model provides a safe and effective pathway for diverting care of low risk patients presenting acutely with chest pain of presumed cardiac origin to the outpatient department. The low rate of re-presentations without any adverse events demonstrates the safety of this approach. Clinical outcomes within the 3 months were excellent with only 1 patient requiring revascularisation. Furthermore, there were significant service efficiencies delivered with benefits evidenced across both primary and secondary care.

\section{THE DIAGNOSIS OF STABLE ANGINA: IS THERE STILL A ROLE FOR THE RAPID ACCESS CHEST PAIN CLINIC?}

${ }^{1}$ Jonathan Batty ${ }^{*},{ }^{2}$ fftikhar Haq. ${ }^{1}$ Newcastle University; ${ }^{2}$ Newcastle Upon Tyne Hospitals NHS Foundation Trust; *Presenting Author

\subsection{6/heartjnl-2016-309890.82}

Introduction Stable angina is diagnosed on the basis of clinical assessment and diagnostic testing. Angina-type pain is defined by: (i) constricting discomfort in the chest, neck, shoulders, jaw, or arms, (ii) precipitated by physical exertion, and (iii) relieved by rest or glyceryl trinitrate (GTN). Typicality of these symptoms, in addition to risk factor burden, is used to estimate the pre-test probability of coronary artery disease, guiding investigation and management. However, it remains unclear whether Rapid Access Chest Pain Clinic (RACPC) assessment provides advantages to that in primary care. We studied patients with suspected stable angina, to evaluate concordance between GP and cardiologist findings, and identify barriers to diagnosis in primary care.

Methods A database of all patients reviewed in a high-volume, cardiologist-led RACPC was prospectively maintained, from Jul 2012-Jan 2015. Standardised GP referral proformas were used to code typicality of angina pain ( 3 features -- typical angina; 2 features - atypical angina; 0 or 1 feature - non-angina), and missing data identified. Concordance between GP and cardiologist assessment was ascertained using Cohen's kappa (K) and Bland-Altman plots. Age, sex, typicality of angina, presence of risk factors (smoking, diabetes or hyperlipidaemia) and ECG criteria (presence of Q-waves or ST/T changes) were used to estimate GP and cardiologist-calculated pre-test probabilities, compared using Student's $t$-test. Thematic analysis of free-text clinical details was performed, to explore reasons for missing data. All data are presented: mean [SD].

Results Data regarding $n=1928$ referrals were available. After exclusion of cases for which referral was via non-structured or incomplete letter, 1634 cases were included in the final analysis. Agreement between GP and cardiologist in typicality of angina was poor $(723 / 1634,44.2 \% ; \mathrm{K}=0.235 p$ $<0.001$; Table 1, Figure 1). Pre-test probability calculated using GP assessment was greater than that assessed by cardiologist (54.7 [25.0] vs. 40.9\% [22.2]; $p<0.001)$, although a strong positive correlation was observed $\left(\mathrm{R}^{2}=0.56, p<\right.$ 0.001 ; Figure 2). GP referral data regarding character of chest pain were missing in $n=10(0.6 \%)$. Thematic analysis demonstrated this was primarily due to non-pain related symptoms, predominantly breathlessness. No data regarding relationship to exertion were available in $n=57$ (3.5\%), due 


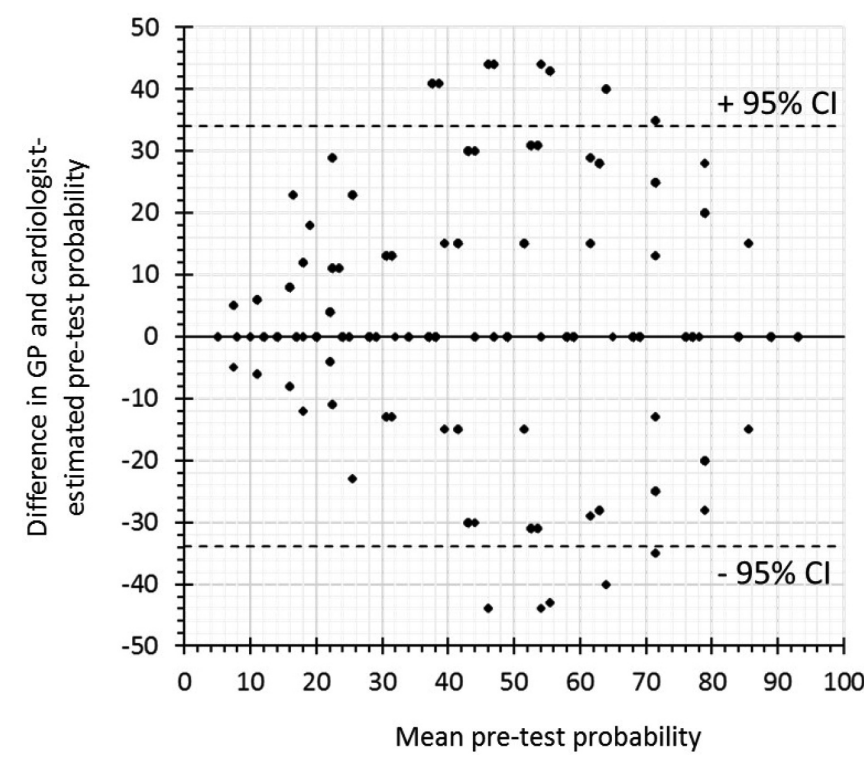

Abstract 82 Figure 1 Bland-Altman plot of agreement between GP and cardiology-estimated pre-test probability for coronary artery disease

to short duration of pain. No data regarding relationship to rest or GTN were available in $n=475$ (29.1\%), attributable to the referrer only considering GTN, which was often only prescribed at the point of referral.

Abstract 82 Table 1 Concordance between GP and RACPC diagnosis of anginal-type chest pain

\begin{tabular}{lllll}
\hline & & \multicolumn{3}{c}{ RACPC diagnosis } \\
\hline \multirow{4}{*}{ GP diagnosis } & Typical angina & Atypical angina & Non-angina \\
\cline { 3 - 5 } & Typical angina & 303 & 140 & 18 \\
& Atypical angina & 324 & 335 & 64 \\
& Non-angina & 141 & 224 & 85 \\
\hline
\end{tabular}

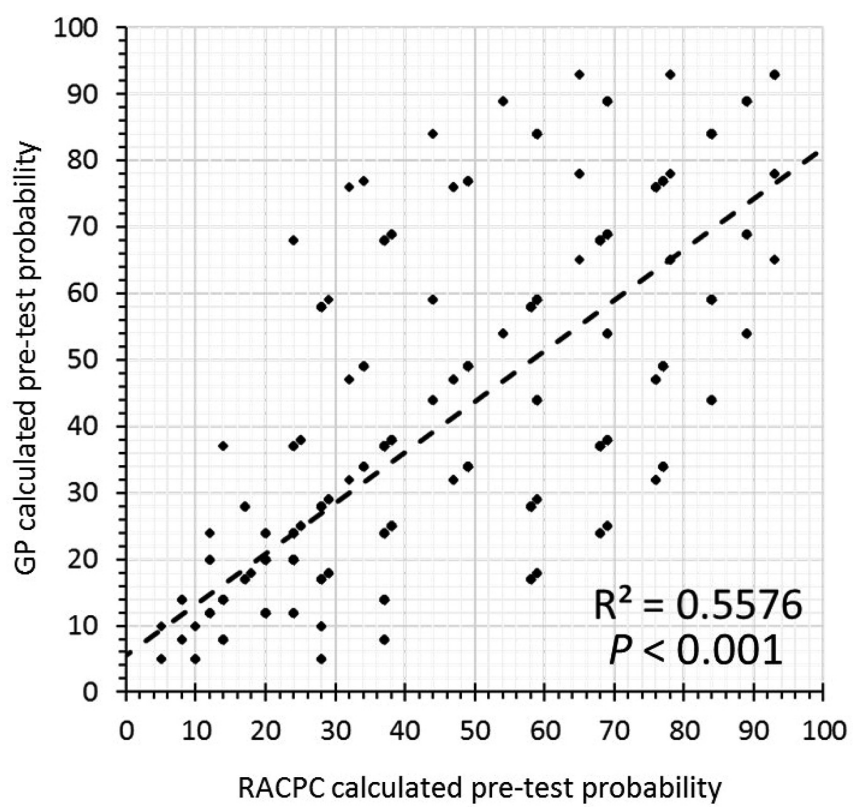

Abstract 82 Figure 2 Correlation between GP and cardiologyestimated pre-test probability for coronary artery disease
Conclusions GP and cardiologist-led diagnosis of stable angina is discordant; the pre-test probability is overestimated in primary care, which would have significant implications for subsequent diagnostic investigation. The RACPC remains an essential service for patients with suspected stable angina.

\section{SELF-ASSESSMENT OF CARDIOVASCULAR RISK: PUBLIC USE OF THE ONLINE JBS3 HEART AGE TOOL}

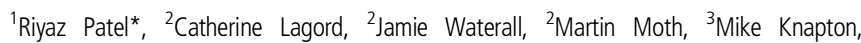
'John Deanfield. ' UCL; ${ }^{2}$ Public Health UK; ${ }^{3}$ British Heart Foundation; * Presenting Author

\subsection{6/heartjnl-2016-309890.83}

Background The JBS3 risk calculator offers a novel approach to cardiovascular risk communication by estimating a "heart age". A modified version of this tool was introduced online to broaden access to personalized risk assessment to the general population and encourage participation in the NHS Health Check programme. This study reports on its early uptake and the profiles of those who used it.

Methods The JBS3 tool estimates a"heart age", through multivariable modelling which is referenced to someone of the same age, gender and ethnicity with optimal risk factors. Between February and July 2015, user data collected from the NHS Choices website (https://www.nhs.uk/tools/pages/heartage. aspx), where the tool was hosted, were analyzed anonymously using standard analytic packages.

Results The online tool was accessed 1.4 million times in the first 5 months, with increased activity following limited media coverage. Of the 575,782 users completing the data journey with a valid"heart age" result, their demographic and risk factor profiles broadly resembled the population of England, although both younger users and males (60\%) were over represented. Almost 50\% and $79 \%$ did not know or enter their blood pressure or cholesterol values, respectively (Figure 1). Estimated"heart age" was higher than chronological age for $79 \%$ of all users, but also for $69 \%$ of younger users under 40 years who are at low 10-year risk and not invited for NHS Health Checks (Figure 2).

Conclusions/interpretation These data suggest a high level of public interest in self-assessment of cardiovascular risk when an easily understood metric is used, although a large number

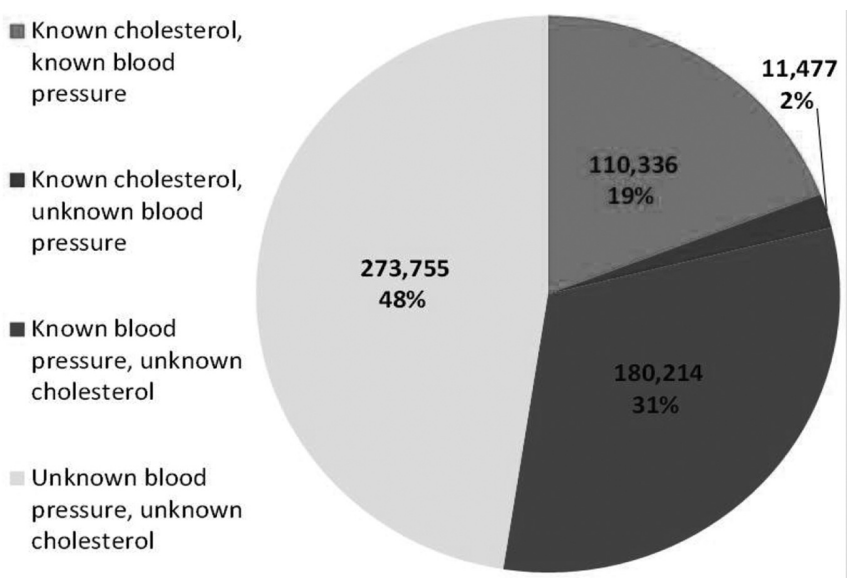

Abstract 83 Figure 1 Proportion of users who were unaware of their blood preasure and/or cholesterol 